\title{
Micro-Transfer Printing of Lithium Niobate on Silicon Nitride
}

\author{
Tom Vanackere ${ }^{(1,2,4)}$, Maximilien Billet ${ }^{(1,2,4)}$, Camiel Op de Beeck ${ }^{(1,2)}$, Stijn Poelman ${ }^{(1,2)}$, \\ Gunther Roelkens ${ }^{(1,2)}$, Stéphane Clemmen ${ }^{(1,2,3,4)}$, Bart Kuyken ${ }^{(1,2)}$
}

(1) Photonics Research Group, Department of Information Technology, Ghent University-IMEC, Technologiepark 126, 9052 Ghent, Belgium Tom.Vanackere@ugent.be

(2) Center for Nano- and Biophotonics, Ghent University, 9052 Ghent, Belgium

(3) Laboratoire d'Information Quantique, Université Libre de Bruxelles, 1050 Bruxelles, Belgium

(4) OPERA-Photonique CP 194/5, Université Libre de Bruxelles (ULB), Bruxelles, Belgium

Abstract Successful micro-transfer printing of lithium niobate on a silicon nitride platform is demonstrated. A proof of concept electro-optical modulator is fabricated using this hybrid integration method which shows a half-wave voltage-length product $V_{\pi} L_{\pi}=5.5 \mathrm{Vcm}$ and insertion losses of $7 \mathrm{~dB}$.

\section{Introduction}

Modulators are an important element for any photonics platform. For silicon nitride (SiN) photonics, optical modulation is often achieved using the thermo-optic effect. But these modulators come with disadvantages in speed and power efficiency. A popular alternative is electro-optic modulation which is able to provide pure phase modulation at much higher speeds. The $\chi^{(2)}$ response in SiN itself is rather weak ${ }^{[1]}$, therefore to access electrooptic modulation on an integrated SiN platform, a hybrid solution has to be found, such as the co-integration of barium titanate (BTO) ${ }^{[2]}$ or lead zirconate titanate $(\mathrm{PZT})^{[3]}$ thin films with the SiN photonic platform. Lithium niobate $(\mathrm{LN})$ remains the gold standard when it comes to electro-optic modulation. Thin films of $L N$ have been used as a standalone integrated platform ${ }^{[4]-[6]}$ or have been used for hybrid integration with both silicon (Si) and SiN integrated platforms ${ }^{[5],[7],[8]}$. Although bonding has been the preferred method for hybrid integration, it has several disadvantages with respect to material use and the co-integration with other hybrid material platforms. Micro-transfer printing on the contrary allows for the integration of micrometer sized material sections or even fully pre-processed devices ${ }^{[9],[10]}$ onto a photonic chip with sub-micrometer precision. This ensures efficient use of the transfer printed material and enables the co-integration with other hybrid material platforms on the same chip. Here we present the first demonstration of micro-transfer printed layers of thin film LN onto a SiN integrated platform. In these hybrid structures the light is guided by the patterned SiN structures while a significant portion of the mode resides in the LN slab on top. We also show as proof of concept an electro-optical modulator with a half-wave voltage-length prod- uct $V_{\pi} L_{\pi}=5.5 \mathrm{Vcm}$ and insertion losses of $7 \mathrm{~dB}$. We will first discuss the fabrication of the device after which both the passive and modulation characteristics are examined.

\section{Micro-Transfer printing of Lithium Niobate on Silicon Nitride}

In micro-transfer printing, a source and a target wafer are first prepared separately before the actual hybrid integration can take place. The main elements of the fabrication process are shown schematically in figure 1 . The source sample is a $300 \mathrm{~nm}$ thin film LN sample purchased commercially from NanoLN. Here, the wafer consists of a $L N$ substrate with a $2 \mu \mathrm{m}$ buffer oxide layer and a $300 \mathrm{~nm}$ LN device layer. First, a chromium $(\mathrm{Cr})$ hard mask is defined using contact UV lithography and lift-off techniques. The pattern is then transferred into the LN layer with a dry Reactive Ion Etching (RIE) process creating the coupon structure shown in figure $1(\mathrm{a}, \mathrm{j})$. This is a rectangular section of $60 \mu \mathrm{m}$ by $1 \mathrm{~mm}$ connected to the rest of the sample through thin tethers. The extraordinary axis of the $\mathrm{LN}$ crystal is aligned along the short side of the rectangle. The sample is subsequently dipped in hydrofluoric acid to underetch the structure by selectively etching away the oxide layer underneath (b). The goal of the tethers is to support the coupon allowing it to remain suspended after the release etch. After the release etch the sample is dried using Critical Point Drying to avoid the capillary forces associated with standard drying.

In parallel the target sample is prepared. An LPCVD SiN wafer purchased from LioniX is patterned with waveguides, grating couplers and Mach-Zehnder interferometers based on Multimode Interference (MMI) splitters using e-beam 


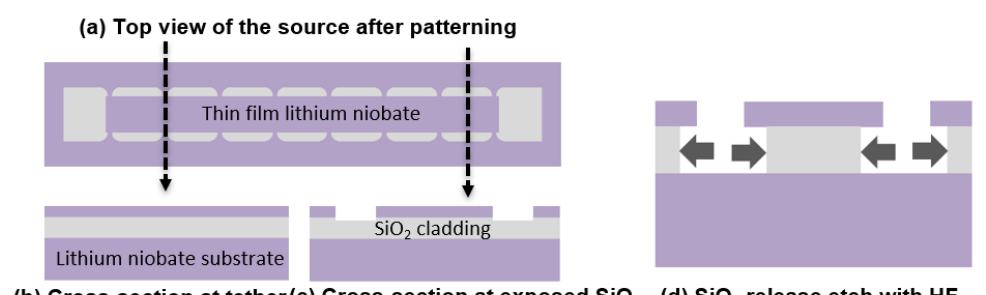

$\begin{array}{lll}\text { (b) Cross-section at tether (c) Cross-section at exposed } \mathrm{SiO}_{2} & \text { (d) } \mathrm{SiO}_{2} \text { release etch with } \mathrm{HF}\end{array}$

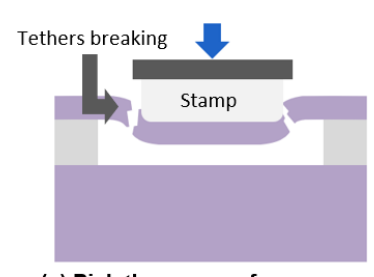

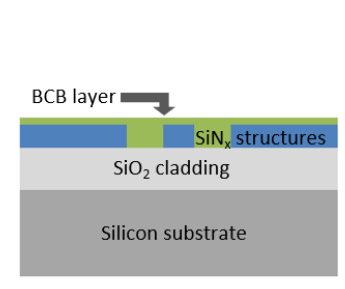

(f) Prepare target sample

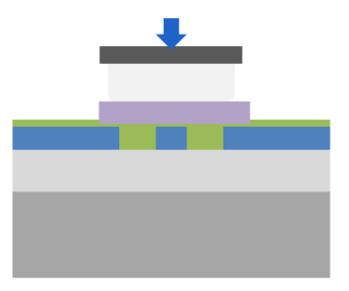

(g) Print the coupon on target

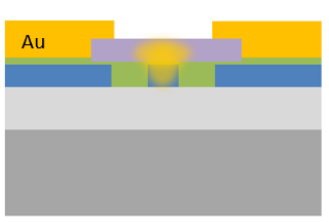

(i) Hybrid mode overlap

(e) Pick the coupon from source

Fig. 1: Schematic representation of the micro-transfer printing process flow.

lithography and RIE. The SiN layer is $300 \mathrm{~nm}$ thick on a $3.3 \mu \mathrm{m}$ thick layer of buffer oxide and a silicon substrate. Next, a layer of approximately $100 \mathrm{~nm}$ of Benzocyclobutene (BCB) polymer is spin-coated on top of the target sample after which it is pre-baked to prepare it for the printing process. The source and target samples are then carefully aligned in the transfer-printing tool. An elastomeric stamp (Polydimethylsiloxane) is slowly pressed against the target coupon after which it is quickly elevated. During the rapid upwards acceleration, the adhesion to the elastomer stamp is sufficiently strong to cause the thin tethers to break and the coupon to detach from the source sample. It is then moved to the target where it is aligned and pressed against the sample. When slowly retracting the stamp, the adhesion force is weaker, allowing the coupon to stick to the BCB on the target. It can be scaled up to print arrays of coupons automatically using digital pattern recognition to allow for wafer scale hybrid integration. The alignment accuracy of printed single coupons can be lower than $0.5 \mu \mathrm{m}$ in stateof-the-art micro-transfer printing tools. This process also allows the source and target wafers to be prepared separately, thus avoiding the need to process contaminating materials such as $L N$ in the same fab.

\section{Passive properties}

To evaluate the properties of the LN coupons, Mach-Zehnder interferometer (MZI) structures were fabricated. While the MZI has arms of the same length, by transfer printing a $L N$ coupon onto one of the arms, an unbalanced MZI is created. The measured signal is normalized using a similar structure without any coupons to eliminate all losses associated with the grating cou- plers and MMls. The results of a device using a $\mathrm{SiN}$ waveguide of $1.4 \mu \mathrm{m}$ width are shown in figure 2. The plot indicates a typical MZI pattern with a Free Spectral Range (FSR) of approximately $12 \mathrm{~nm}$ and an Extinction Ratio (ER) of $12.0 \mathrm{~dB}$. Assuming the loss in the couponless MZI arm is zero, the loss in the arm with a coupon can be calculated to be around $4 \mathrm{~dB}$. FDTD simulations predict transition losses of around $2.0 \mathrm{~dB} /$ facet, indicating the propagation losses remain minimal. Measurements on structures with slightly different geometries result in similar conclusions.

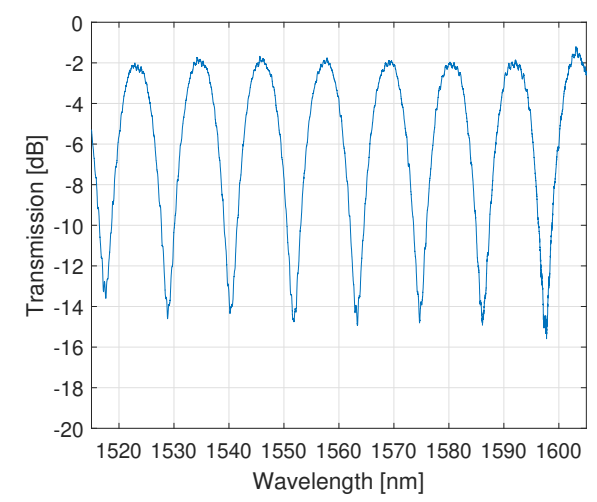

Fig. 2: Transmission spectrum of an integrated MZI with a micro-transfer printed LN coupon on one arm of the interferometer. No electrodes or bias voltage is present here.

\section{Hybrid Lihium Niobate on Silicon Nitride} electro-optic modulator

After performing the passive measurements, electrodes are added to the MZI structures such that the phase in one arm can be changed using the electro-optic effect of the LN coupon. The electrodes are $500 \mathrm{~nm}$ thick, $0.9 \mathrm{~mm}$ long and have a $6.5 \mu \mathrm{m}$ gap. The results of the electrooptic measurements are shown in figure 3 . The measurement data was normalized with the insertion loss, which was estimated to be around 
(a)

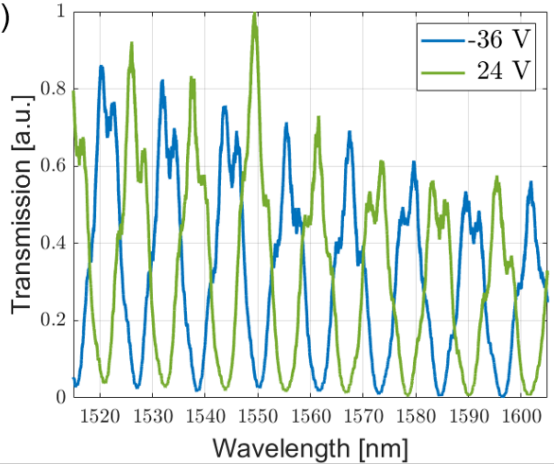

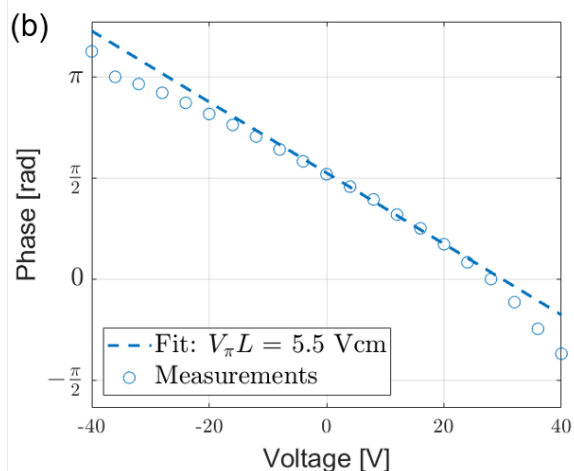

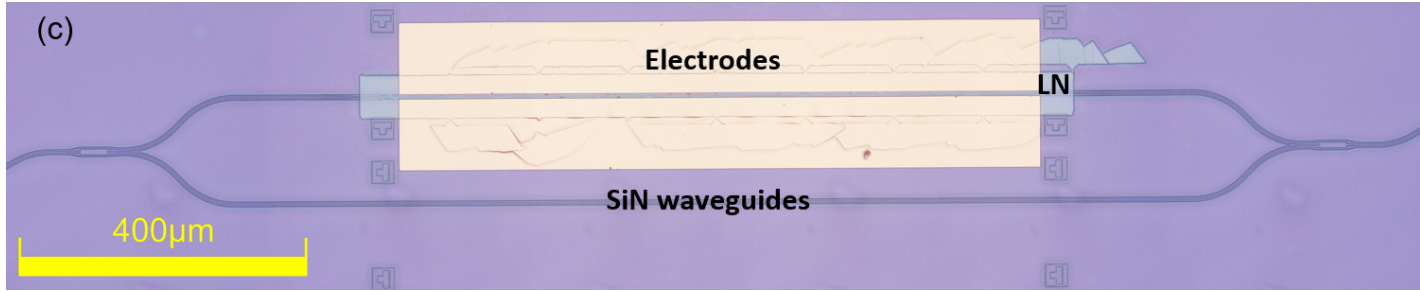

Fig. 3: (a) Transmission spectra at two different bias voltages for an MZI with a micro-transfer printed LN coupon on one arm of the interferometer. (b) Phase difference between the light in both arms as a function of the bias voltage, extracted from measurements at a wavelength of $1550 \mathrm{~nm}$. (c) Microscope image of the whole MZI used for electro-optical modulation.

$7 \mathrm{~dB}$ at $1550 \mathrm{~nm}$, a large portion of these losses are due to imperfect MMI splitters and could be improved later.

It is observed that the signal peaks have a dissimilar shape compared to the measurement data without electrodes. This could be the result of some reflection or some defect introduced during metalisation. However the two curves in the figure measured at a bias voltage of $-36 \mathrm{~V}$ and $24 \mathrm{~V}$ clearly show the ability of this modulator to shift the resonances by a significant amount. Figure 3 (b) shows the phase difference between the light in both arms as a function of the bias voltage measured and fitted to the results at $1550 \mathrm{~nm}$. From this we estimate a half-wave voltage-length product $V_{\pi} L_{\pi}=5.5 \mathrm{Vcm}$. This is slightly better than the simulated value of $6.0 \mathrm{Vcm}$, this could be explained by a small misalignment of the electrodes resulting in stronger fields but also higher than intended losses. The linearity of this phase difference even when the bias voltage changes sign is a clear indicator that the modulation is due to the electro-optical effect, which is promising for high speed operation.

\section{Conclusions and future prospects}

We demonstrated the first devices using microtransfer printed thin film LN on a SiN integrated platform. A simple MZI device was used to measure both the losses of the coupon and the halfwave voltage-length product of a modulator which both show promising results that resemble the simulated values. We plan to further optimise the transfer printing process to improve the coupon quality and yield while also looking into printing longer coupons. More research will also be done to reduce the transition loss at the interface using for example tapered structures. We believe this process methodology allows us to combine the strong electro-optical and nonlinear properties of $L N$ with various passive photonic platforms, as well as with other material platforms such as III-V gain materials, enabling the creation of complex, multi-functional devices on a single photonic chip.

\section{Acknowledgements}

Tom Vanackere and Camiel Op de Beeck are $\mathrm{PhD}$ fellows of the Research Foundation Flanders (FWO) under respective grant numbers $11 \mathrm{~F} 5320 \mathrm{~N}$ and $1 \mathrm{~S} 54418 \mathrm{~N}$. Stéphane Clemmen is a research associate of the Fonds de la Recherche Scientifique - FNRS. We also thank the European Research Council (ERC) for funding in the context of the ELECTRIC project.

\section{References}

[1] M. A. Porcel, J. Mak, C. Taballione, V. K. Schermerhorn, J. P. Epping, P. J. van der Slot, and K.-J. Boller, "Photoinduced second-order nonlinearity in stoichiometric silicon nitride waveguides", Opt. Express, vol. 25, no. 26, pp. 33 143-33159, 2017.

[2] J. E. Ortmann, F. Eltes, D. Caimi, N. Meier, A. A. Demkov, L. Czornomaz, J. Fompeyrine, and S. Abel, "Ultra-low-power tuning in hybrid barium titanate-silicon nitride electro-optic devices on silicon", ACS Photonics, vol. 6, no. 11, pp. 2677-2684, 2019. 
[3] K. Alexander, J. P. George, J. Verbist, K. Neyts, B. Kuyken, D. Van Thourhout, and J. Beeckman, "Nanophotonic pockels modulators on a silicon nitride platform", Nature Communications, vol. 9, no. 1, p. 3444, 2018.

[4] C. Wang, M. Zhang, X. Chen, M. Bertrand, A. ShamsAnsari, S. Chandrasekhar, P. Winzer, and M. Lončar, "100-ghz low voltage integrated lithium niobate modulators", in Conference on Lasers and Electro-Optics, Optical Society of America, 2018.

[5] A. Honardoost, K. Abdelsalam, and S. Fathpour, "Rejuvenating a versatile photonic material: Thin-film lithium niobate", Laser \& Photonics Reviews,

[6] J. Lu, J. B. Surya, X. Liu, A. W. Bruch, Z. Gong, Y. Xu, and $\mathrm{H}$. X. Tang, "Periodically poled thin-film lithium niobate microring resonators with a second-harmonic generation efficiency of $250,000 \% /$ w", Optica, vol. 6 , no. 12 , pp. 1455-1460, 2019.

[7] L. Chang, M. H. P. Pfeiffer, N. Volet, M. Zervas, J. D. Peters, C. L. Manganelli, E. J. Stanton, Y. Li, T. J. Kippenberg, and J. E. Bowers, "Heterogeneous integration of lithium niobate and silicon nitride waveguides for waferscale photonic integrated circuits on silicon", Opt. Lett., vol. 42, no. 4, pp. 803-806, 2017.

[8] M. He, M. Xu, Y. Ren, J. Jian, Z. Ruan, Y. Xu, S. Gao, S. Sun, X. Wen, L. Zhou, L. Liu, C. Guo, H. Chen, S. Yu, L. Liu, and X. Cai, "High-performance hybrid silicon and lithium niobate mach-zehnder modulators for $100 \mathrm{gbit} / \mathrm{s}$ and beyond", Nature Photonics, vol. 13, no. 5, pp. 359364, 2019.

[9] B. Haq, S. Kumari, K. Van Gasse, J. Zhang, A. Gocalinska, E. Pelucchi, B. Corbett, and G. Roelkens, "Micro-transfer-printed iii-v-on-silicon c-band semiconductor optical amplifiers", Laser \& Photonics Reviews, vol. 14, no. 7, 2020.

[10] J. Justice, C. Bower, M. Meitl, M. B. Mooney, M. A. Gubbins, and B. Corbett, "Wafer-scale integration of group iii-v lasers on silicon using transfer printing of epitaxial layers", Nature Photonics, vol. 6, no. 9, pp. 610-614, 2012. 